\title{
JOINT POWER CONTROL AND BEAMFORMING FOR CAPACITY IMPROVEMENT IN WIRELESS NETWORKS WITH ANTENNA ARRAYS
}

\author{
F. Rashid-Farrokhi, L. Tassiulas, and K. J. R. Liu \\ Electrical Engineering Department and Institute for Systems Research \\ University of Maryland \\ College Park, MD 20742, USA
}

\begin{abstract}
The interference reduction capability of antenna arrays and the power control algorithms have been considered separately as means to increase the capacity in wireless communication networks. The minimum variance beamformer maximizes the Carrier to Interference Ratio (CIR) when it is employed in the receiver of a wireless link. In a system with omnidirectional antennas, power control algorithms are used to maximize CIR as well. In this paper we consider a system with power control capabilities which uses antenna arrays at receivers. An iterative algorithm is proposed to jointly update the transmission powers and the beamformer weights so that it converge to the jointly optimal beamforming and transmission power vector. The algorithm is distributed and uses only local interference measurements. In an uplink transmission scenario it is shown how base assignment can be incorporated in addition to beamforming and power control such that a globally optimum solution is obtained. The network capacity increase and the saving in mobile power achieved by beamforming are evaluated through numerical study.
\end{abstract}

\section{INTRODUCTION}

Cochannel interference is one of the main impairments that degrades the performance of a wireless link. Power control and antenna beamforming are two approaches for improving the performance in wireless networks by appropriately controlling the cochannel interference.

In power control, the transmitter powers are constantly adjusted by increasing if the Signal to Noise Ratio (SNR) is low and decreasing if the SNR is high, so that the quality of weak links is improved. Receivers employing antenna arrays may adjust their beam patterns such that they have maximum gain towards the directions of their transmitters and minimum gain towards the other directions so that the aggregate interference power is minimized.

This work is supported in part by the NSF NYI Award MIP9457397, NSF grant NCR-940641.5, CAREER award NCR9502614, and AFOSR 95-1-0061.
Previous work, discussed later in more detail, addressed separately the problems of power control for optimal interference balancing and beamforming to maximize the SNR. In this paper we consider the joint problem. We consider a set of cochannel links, the receivers of which employ antenna arrays, e.g., a set of cochannel uplinks in a cellular network. An algorithm is provided for computing the transmission powers and the beamforming weight vectors, such that a target SNR is achieved for each link (if it is achievable) with minimal total transmission power. The algorithm is decentralized and amenable to a distributed implementation. In the case that each transmitter has multiple options regarding which receiver to engage, e.g. each mobile may select its base station among a set of possible options, the algorithm easily extends to find the joint optimum power, base station and beamforming.

The application of antenna arrays has been proposed in [1] to increase network capacity in CDMA systems. This work was based on equal received power from all users in base stations. In [2], [3] centralized power control schemes have been proposed to balance the Carrier to Interference Ratio (CIR) or maximize the minimum CIR in all links. These algorithms need global information about all link gains and powers. The distributed power control algorithm which uses only local measurements of CIR was presented in [4], [5]. In $[6]$ and [7] the combined base station assignment and power allocation was used to increase uplink capacity in wireless communication networks. In those works it was shown that if there exists at least one feasible base station assignment, the proposed algorithms will converge to the optimal base station assignment and power allocation in the sense that it minimizes the interference.

The paper is organized as follows. In Section 2, we describe the system model and existing power control algorithms. Section 3 considers the beamforming problem in a network of users. In Section 4, we consider power allocation and beamforming as a joint problem. An iterative algorithm for the joint problem is presented which converges to the optimal solution, i.e., the allocated powers are minimum among all sets of 
feasible power allocations. In Section 5, we consider an uplink scenario where in addition to power control and beamforming base station assignment is performed as well.

In Section 6 a simulation study is done. The simulation results show that using antenna arrays with four elements, the speed of convergence is increased about three times and the capacity is increased about five times compared to the case of joint power control and base station assignment with omnidirectional antennas.

\section{SYSTEM MODEL AND POWER CONTROL PROBLEM}

A set of $M$ transmitter-receiver pairs which share the same channel is considered. The shared channel could be a frequency band in FDMA, a time slot in TDMA, or even CDMA spreading codes. The link gain between transmitter $i$ and receiver $j$ is denoted by $G_{i j}$, and the $i$ th transmitter power by $P_{i}$. For an isotropic antenna with unity gain in all directions, the signal power received at receiver $j$ from transmitter $i$ is $G_{i j} P_{i}$. It is assumed that transmitter $i$ communicates with receiver $i$. Hence the desired signal at receiver $i$ is equal to $G_{i i} P_{i}$, while the interfering signal power from other transmitters to receiver $i$ is $I=\sum_{j \neq i} G_{j i} P_{j}$. The CIR at the $i$ th receiver is given by:

$$
\Gamma_{i}=\frac{G_{i i} P_{i}}{\sum_{j \neq i} G_{j i} P_{j}+N_{i}},
$$

where $N_{i}$ is noise power at the $i$ th receiver. The quality is acceptable if $\Gamma_{i}$ is above a certain threshold $\gamma_{0}$. That is,

$$
\Gamma_{i} \geq \gamma_{0}, \quad 1 \leq i \leq M,
$$

or in matrix form

$$
\left[I-\gamma_{0} F\right] \mathbf{P} \geq \mathbf{u}
$$

where $I$ is an $M \times M$ identity matrix, $\mathbf{u}$ is an elementwise positive vector with elements $u_{i}$ defined as

$$
u_{i}=\frac{\gamma_{0} N_{i}}{G_{i i}}, \quad 1 \leq i \leq M
$$

and

$$
F_{i j}=\left\{\begin{array}{lll}
0 & \text { if } j=i \\
\frac{G_{j i}}{G_{i i}}>0 & \text { if } j \neq i
\end{array} .\right.
$$

The CIR threshold $\gamma_{0}$ is achievable if there exists at least one solution vector $\mathbf{P}$ that satisfies (1). The power control problem now is as follows:

$$
\begin{array}{cl}
\text { minimize } & \sum P_{i}, \\
\text { subject to } & {\left[I-\gamma_{0} F\right] \mathbf{P} \geq \mathbf{u} .}
\end{array}
$$

It can be shown that if the spectral radius of $F$ is less than $\frac{1}{\gamma_{0}}$, the matrix $I-\gamma_{0} F$ is invertible and positive [8]. In this case the power vector

$$
\hat{\mathbf{P}}=\left[I-\gamma_{0} F\right]^{-1} \mathbf{u}
$$

solves the optimization problem.

A centralized power control algorithm ([3]) solves (2) by using all link gains in the network and noise levels at receivers. In [4], [5] a decentralized solution to the power control problem is proposed that achieves (2) by performing the following iteration

$$
\mathbf{P}^{n+1}=\gamma_{0} F \mathbf{P}^{n}+\mathbf{u}
$$

and it has been shown that it converges to the optimal power allocation, i.e., $\hat{\mathbf{P}}=\lim _{n \rightarrow \infty} \mathbf{P}^{n}$.

\section{ANTENNA ARRAY AND BEAMFORMING}

Assume that antenna arrays are deployed at receivers, and each array consists of $K$ elements. Consider a cochannel set consisting of $M$ transmitter and receiver pairs and denote the signal from the $j$ th source by $s_{j}(t)$ and its power by $P_{j}$. The received signal at the $i$ th receiver can be written as follows

$$
\mathbf{x}_{i}(t)=\sum_{j=1}^{M} \sqrt{P_{j}} s_{j}(t) \mathbf{a}_{j i}+\mathbf{n}_{i}(t),
$$

where $K \times 1$ vector $\mathbf{a}_{j i}$ is called the spatial signature or array response of the $i$ th array to the $j$ th source, and $\mathbf{n}_{i}(t)$ is the thermal noise vector at the input of this array. We can write the output of the beamformer at the $i$ th receiver as

$$
\mathbf{e}_{i}=\mathbf{w}_{i}^{H} \mathbf{x}_{i}
$$

where $\mathbf{w}_{i}$ and $\mathbf{x}_{i}$ are the beamforming weight vector and the received signal vector at the $i$ th receiver, respectively. The average output power is given by

$$
\begin{aligned}
\mathcal{E}_{i} & =\mathrm{E}\left\{\mathbf{w}_{i}^{H} \mathbf{x}_{i} \mathbf{x}_{i}^{H} \mathbf{w}_{i}\right\} \\
& =\sum_{j} P_{j} G_{j i} \mathbf{w}_{i}^{H} \mathbf{a}_{j i} \mathbf{a}_{j i}^{I I} \mathbf{w}_{i}+N_{i} \mathbf{w}_{i}^{H} \mathbf{w}_{i},
\end{aligned}
$$

where $N_{i}$ is the noise power at the input of each array element. In order to minimize the interference, we minimize $\mathcal{E}_{i}$ subject to maintaining unity gain at the direction of the desired signal. It can be shown that the optimal weight vector is given by [9]

$$
\hat{\mathbf{w}}_{i}=\frac{\Phi_{i n}^{-1} \mathbf{a}_{i i}}{\mathbf{a}_{i i}^{H} \Phi_{i n}^{-1} \mathbf{a}_{i i}},
$$

where

$$
\Phi_{i n}=\sum_{j \neq i} P_{j} G_{j i} \mathbf{a}_{j i} \mathbf{a}_{j i}^{H}+N_{i} I
$$


The maximum CIR at the $i$ th receiver is given by

$$
\Gamma_{i}=\frac{P_{i} G_{i i}}{\sum_{j \neq i} G_{j i} G_{a_{i}}\left(\hat{\mathbf{w}}_{i}, \mathbf{a}_{j i}\right) P_{j}+N_{i} \hat{\mathbf{w}}_{i}^{H} \hat{\mathbf{w}}},
$$

where $G_{a_{i}}\left(\mathbf{w}_{i}, \mathbf{a}_{j i}\right)=\mathbf{w}_{i}^{H} \mathbf{a}_{j i} \mathbf{a}_{j i}^{H} \mathbf{w}_{i}$ is the $i$ th antenna gain towards the $j$ th source. Here we use the fact that the gain at the direction of interest is unity, i.e, $\mathbf{w}_{i}^{H} \mathbf{a}_{i i}=1$.

\section{JOINTLY OPTIMAL POWER CONTROL AND BEAMFORMING}

In the joint power control and beamforming problem the objective is to find the optimal weight vector and power allocations such that the required CIR is achieved by all links, while each transmitter keeps the transmission power at the minimum required level to reduce the interference to other users. The optimization problem now is defined as

$$
\min _{\mathbf{w}, \mathbf{P}} \sum_{i=1}^{M} P_{i}
$$

subject to $\quad \Gamma_{i} \geq \gamma_{0}, \quad(i=1,2, \ldots, M)$.

This constraint can be presented in matrix form as:

$$
\left[I-\gamma_{0} F^{W}\right] \mathbf{P} \geq \mathbf{u},
$$

where

$$
\left[F^{W}\right]_{i j}=\left\{\begin{array}{ll}
0 & \text { if } j=i \\
\frac{G_{j i} G_{a_{i}}\left(\mathbf{w}_{i}, \mathbf{a}_{j i}\right)}{G_{i i}}>0 & \text { otherwise }
\end{array},\right.
$$

and $\mathbf{u}$ is an element-wise positive vector with elements $u_{i}$ defined as

$$
u_{i}=\frac{N_{i} \mathbf{w}_{i}^{H} \mathbf{w}_{i}}{G_{i i}}, \quad(i=1,2, \ldots, M) .
$$

Assume that there is a set of weight vectors $\mathbf{W}=$ $\left\{\mathbf{w}_{1}, \mathbf{w}_{2}, \ldots, \mathbf{w}_{M}\right\}$, for which $\rho\left(F^{W}\right)<\frac{1}{\gamma_{0}}$. The matrix $I-\gamma_{0} F^{W}$ is then invertible and $\mathbf{P}_{w}=\left[I-\gamma_{0} F^{W}\right]^{-1} \mathbf{u}$ minimizes the objective function in the optimization problem for the fixed weight vector set $\mathbf{W}$. For any feasible pair $\left(\mathbf{W}, \mathbf{P}_{w}\right)$, the vector $\mathbf{P}_{w}$, can be computed as the limit of the following iteration

$$
\begin{array}{r}
P_{i}^{n+1}=\gamma_{0} \sum_{k \neq i} \frac{G_{k i} G_{a_{i}}\left(\mathbf{w}_{i}, \mathbf{a}_{k i}\right)}{G_{i i}} P_{k}^{n}+\frac{\gamma_{0} N_{i} \mathbf{w}_{i}{ }^{H} \mathbf{w}_{i}}{G_{i i}}, \\
(i=1,2, \ldots, M) .
\end{array}
$$

The above iteration is the same as the distributed power control algorithm (see [4]-[5]), in which the path gain $G_{k i}$ is replaced by the multiplication of the path gain and antenna gain, and the noise power is replaced by the weighted sum of the noise powers at the input of each antenna array. Denote the iteration in (6) as

$$
\mathbf{P}^{n}=m^{w}\left(\mathbf{P}^{n-1}\right) .
$$

Starting from any initial power vector $\mathbf{P}^{0}$, the mapping $m^{w}$ will converge to the optimal power vector $\mathbf{P}_{w}$ which is the fixed point of the mapping, i.e., $\lim _{n \rightarrow \infty} \mathbf{P}^{n}=$ $\mathbf{P}_{w}, \mathbf{P}_{w}=m^{w}\left(\mathbf{P}_{w}\right)$. The objective in joint optimal beamforming and power control problem is to find the beamforming set $W$ among all feasible beamforming sets, in such a way that $\mathbf{P}_{w}$ is minimal.

The level of cochannel interference depends both on the gain between interfering transmitters and receivers, as well as on the level of transmitter powers, i.e., the optimal beamforming vector may vary for different powers. Hence, beamforming and power control should be considered jointly.

Let $(\hat{\mathbf{W}}, \hat{\mathbf{P}})$ be the weight vector and power vector pair which achieves the minimum in (5). In the sequel, we present an iterative algorithm for adjusting $\mathbf{P}$ and W simultaneously. We can show that starting from any arbitrary power vector, it converges to the optimal solution $(\hat{\mathbf{W}}, \hat{\mathbf{P}})$. The iteration steps for obtaining $\left(\mathbf{P}^{n+1}, \mathbf{W}^{n+1}\right)$ given $\left(\mathbf{P}^{n}, \mathbf{W}^{n}\right)$ are as follows:

\section{Algorithm A:}

1. $\mathbf{w}_{i}^{n+1}$ is computed at each receiver $i$ such that the cochannel interference is minimized under the condition of constant gain for the direction of interest, i.e.,

$$
\mathbf{w}_{i}^{n+1}=\arg \min _{\mathbf{w}_{i}}\left\{\sum_{j \neq i} G_{j i} G_{a_{i}}\left(\mathbf{w}_{i}, \mathbf{a}_{j i}\right) P_{j}^{n}+\right.
$$

subject to

$$
\left.N_{i} \mathbf{w}_{i}^{H} \mathbf{w}_{i}\right\},
$$

$$
\mathbf{w}_{i}{ }^{H} \mathbf{a}_{i i}=1,
$$

$$
(i=1,2, \ldots, M),
$$

where $\mathbf{P}^{n}$ is the power vector updated at step $n$.

2. The updated power vector, $\mathbf{P}^{n+1}$, is then obtained by

$$
\begin{aligned}
P_{i}^{n+1}=\gamma_{0} \sum_{k \neq i} \frac{G_{k i} G_{a_{i}}\left(\mathbf{w}_{i}^{n+1}, \mathbf{a}_{k i}\right)}{G_{i i}} P_{k}^{n} & \\
& +\frac{\gamma_{0} N_{i}\left(\mathbf{w}_{i}^{n+1}\right)^{H} \mathbf{w}_{i}^{n+1}}{G_{i i}},
\end{aligned}
$$

that is, by performing one iteration with the mapping $m^{\mathbf{w}_{i}^{n+1}}$ on the power vector $\mathbf{P}^{n}$. holds:

In [10] we have proved that the following theorem

Theorem 1 The sequence $\left(\mathbf{P}^{n}, \mathbf{W}^{n}\right),(n=1,2, \ldots)$ produced by algorithm $A$ starting from an arbitrary power vector $\mathbf{P}^{0}$, converges to the optimal pair $(\hat{\mathbf{P}}, \hat{\mathbf{W}})$. 


\section{JOINT POWER CONTROL, BASE STATION ASSIGNMENT, AND BEAMFORMING}

In the joint power control and base station assignment, a number of base stations are potential receivers of a mobile transmitter. In the optimal power control and base station assignment, the objective is to determine the assignment of users to base stations which minimizes the allocated mobile powers, and the corresponding transmission powers. Iterative algorithms that compute the jointly optimal base station assignment and power allocation were proposed in [6], [7].

In an uplink scenario where mobiles access base stations equipped with antenna arrays, the problem of joint power control and beamforming as well as base station assignment naturally arises. A modification of the iterative algorithm we proposed for the joint power control and beamforming problem can perform base assignment as well. The iterative steps of the algorithm are as follows:

\section{Algorithm B}

1. Each base station in the allowable set of a mobile $i$ minimizes the total interference subject to maintaining unity gain towards the direction of this mobile:

$$
\begin{aligned}
& \begin{array}{l}
\mathbf{w}_{i m}^{n+1}=\arg \min _{\mathbf{w}_{i}}\left\{\sum_{j \neq m} G_{j m} G_{a_{i}}\left(\mathbf{w}_{i}, \mathbf{a}_{j m}\right) P_{j}^{n}\right. \\
\left.\quad+N_{m} \mathbf{w}_{i}^{H} \mathbf{w}_{i}\right\}, \\
\text { subject to } \quad \mathbf{w}_{i}{ }^{H} \mathbf{a}_{i m}=1, \\
i=1,2, \ldots, M, \quad m \in B_{i},
\end{array}
\end{aligned}
$$

where $\mathbf{w}_{i m}^{n+1}$ is the optimal beamforming weight vector at the $m$ th base station for the $i$ th mobile, and $B_{i}$ is the set of allowable base stations for the $i$ th mobile.

2. Each mobile finds the best base station such that the allocated power for the next iteration is minimized:

$$
\begin{aligned}
& b_{i}=\arg \min _{m \in B_{i}}\left\{\gamma_{0} \sum_{k \neq i} \frac{G_{k m} G_{a_{i}}\left(\mathbf{w}_{i m}^{n+1}, \mathbf{a}_{k m}\right)}{G_{i m}} P_{k}^{n}+\right. \\
&\left.\frac{\gamma_{0} N_{m} \mathbf{w}_{i m}^{n+1}{ }^{H} \mathbf{w}_{i m}^{n+1}}{G_{i m}}\right\}, \quad(i=1,2, \ldots, M),
\end{aligned}
$$

where $b_{i}$ is the current assignment for mobile $i$.

3. The mobile $i$ updates the power based on the current beamforming and base station assignment:

$$
P_{i}^{n+1}=\gamma_{0} \sum_{k \neq i} \frac{G_{k b_{i}} G_{a_{i}}\left(\mathbf{w}_{i b_{i}}^{n+1}, \mathbf{a}_{k b_{i}}\right)}{G_{i b_{i}}} P_{k}^{n}+
$$

$$
\frac{\gamma_{0} N_{b_{i}} \mathbf{w}_{i b_{i}}^{n+1} \mathbf{w}_{i b_{i}}^{n+1}}{G_{i b_{i}}}, \quad(i=1,2, \ldots) .
$$

The convergence and optimality of this algorithm can also be proved the same as theorem 1 [10].

\section{SIMULATION RESULTS AND COMPARISONS}

We evaluate the performance of our algorithm by simulating the same CDMA system as in [7]. However, the same methodology can be applied to any wireless network such as TDMA and FDMA. Fig. 1 shows a network with 36 base stations with 400 users randomly distributed in the area $[0.5,6.5] \times[0.5,6.5]$ with uniform distribution. The quality constraint is considered to be 0.0304 . The link gain is modeled as $G_{i j}=1 / d_{i j}^{4}$, where $d_{i j}$ is the distance between base $i$ and mobile $j$. Throughout the simulations, we consider two system setups: in system setup I we use omnidirectional antennas; in system setup II we use antenna array with 4 elements.

Fig. 1(a), illustrates the use of system setup I, in which the mobiles are assigned to the closest base stations. The mobile powers are obtained by iterative fixed assignment power control algorithm as given by (3). In Fig. 2(a), the dashdot curve shows the total mobile power at each iteration. This algorithm converges in about 16 iterations. In Fig. 1(b), using the same system setup, the base station assignment is done by the jointly optimal base station assignment and power control algorithm, and mobiles have the option to select among four closest base stations [7]. The total mobile power is depicted in Fig. 2(a). The dashed curves shows that the total power is slightly less than that of the first algorithm considered in Fig. 1(a). This algorithm converges in about 15 iterations. In Fig. 1(c) we use the system setup II, i.e., the base stations are equipped with 4-element antenna arrays. We apply our joint power control, base station assignment, and beamforming algorithm to the same configuration of users as in Fig. 1(a) and (b). The solid curve in Fig. 2 (a) shows that the total mobile power is an order of magnitude smaller than the previous algorithms. Furthermore, the convergence of this algorithm is faster; it converges in about 5 iterations in our simulation study.

The capacity of the system is defined as the maximum number of users for which there exists a feasible power vector. As the number of users is increased the maximum eigenvalue of the gain matrix, $\rho(F)$, approaches unity and the total sum of mobile power is increased. Using an antenna array with four elements and our algorithm, we can increase the capacity of the network significantly. In Fig. 2(b) the total mobile power versus the number of users is depicted. Using omnidirectional antenna and power control algorithm 
with fixed base assignment, we can tolerate at most 660 users. In the same configuration, using the base station assignment and power control algorithm [7], we can increase the capacity to 800 users. If we use antenna array with four elements, using our algorithm, the network can tolerate 2800 users. Fig. 3 illustrates the base station assignments for the above three cases.

\section{CONCLUSION}

We have introduced the consideration of joint optimal beamforming and power control. We provided an iterative algorithm amenable to distributed implementation which converges to the optimal beamforming and base station assignment if there exists at least one solution to the joint problem. An enhancement of the algorithm that makes it appropriate for joint power control and base assignment as well as beamforming was considered as well.

For performance evaluation of our algorithm a notion of capacity was considered to be the maximum number of transmitters for which there exists a feasible power vector. It was shown that using antenna array at the base stations, the algorithm can improve the capacity of networks significantly in terms of the number of users that can be supported. When we have the same configuration of users, the use of 4-element antenna arrays in the base stations and our algorithm significantly reduces the mobile power by almost an order of magnitude, which is very critical in terms of battery life in mobile sets. On the other hand, it speeds up the convergence compared to the systems with omnidirectional antennas.

\section{REFERENCES}

[1] A. F. Naguib, A. Paulraj, and T. Kailath, "Capacity improvement with base-station antenna arrays in cellular CDMA," IEEE Trans. Vehicular Technol., vol. 43, No. 3, Aug. 1994.

[2] R. W. Nettleton and H. Alavi, "Power control for spread spectrum cellular mobile radio system," in Proc. IEEE Vehicular Technol. Conf., VTC-83, 1983.

[3] S. Grandhi, R. Vijayan, D.J. Goodman, and J. Zander, "Centralized power control for cellular radio systems," IEEE. Trans. Vehicular Tech., vol. 42, Nov. 1993.

[4] J. Zander, "Distributed cochannel interference control in cellular radio systems," IEEE Trans. Vehicular Technol., vol. 41, Aug. 1992.

[5] S. A. Grandhi, R. Vijayan, and D. J. Goodman, "A distributed algorithm for power control in cellular radio systems," in Proc. 30th Allerton Conf. Commun. Control and Computing, Moticello, IL. Sept. 1992.
[6] R. Yates and C. Y. Huang, "Integrated power control and base station assignment," IEEE Trans. Vehicular Technol., vol. 44, No. 3, Aug. 1995.

[7] S. V. Hanly, "An algorithm for combined cell-site selection and power control to maximize cellular spread spectrum capacity," IEEE J. Select. Areas Commun., Vol. 13, No. 7, Sept. 1995.

[8] F. R. Gantmacher, "The Theory of Matrices,", vol. 2, Chelsea, New York, 1990.

[9] R. A. Monzingo and T. W. Miller, Introduction to Adaptive Arrays. New York, Wiley, 1980.

[10] F. Rashid-Farrokhi, L. Tassiulas, K.J.R. Liu, "Joint optimal power control and beamforming in wireless networks using antenna arrays," ISR technical report, Institute for System Research, University of Maryland at College Park, Aug. 1996.

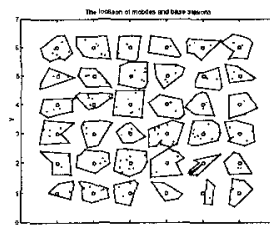

(a)

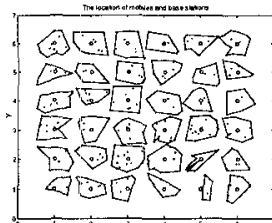

(b)



(c)
Figure 1: Mobile and base stations locations for 400 users; (a) traditional assignment; (b) optimal base station and power control; (b) optimal base station, beamforming and power control.

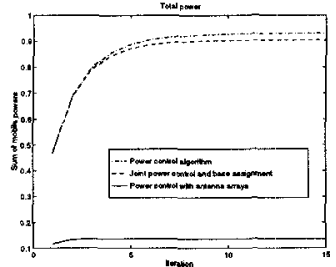

(a)

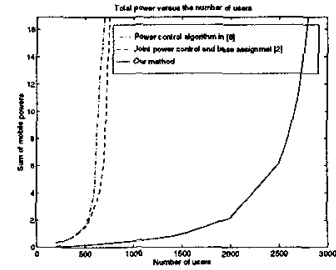

(b)
Figure 2: (a) The total mobile powers versus the iteration number; (b) the total mobile powers versus the number of users.



(a)

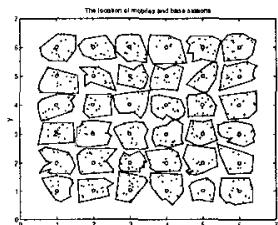

(b)

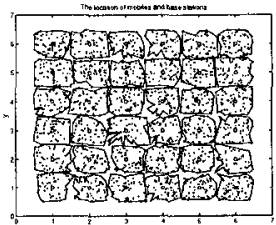

(c)
Figure 3: Mobile and base stations locations; (a) traditional assignment with 660; (b) optimal base station and power control with 800; (b) optimal base station, beamforming and power control with 2800 users. 